\title{
DNA breathers and cell dynamics
}

\author{
Nikitiuk A.S. ${ }^{* 1}$, Korznikova E.A. ${ }^{\dagger 2}$, Dmitriev S.V. ${ }^{\ddagger 2,3}$, Naimark O.B. ${ }^{\$ 1}$ \\ ${ }^{1}$ Institute of Continuous Media Mechanics Ural Branch of RAS, Perm, Russia \\ ${ }^{2}$ Institute for Metals Superplasticity Problems RAS, Ufa, Russia \\ ${ }^{3}$ National Research Tomsk State University, Tomsk, Russia
}

\begin{abstract}
Molecular-morphological signs of oncogenesis can be linked to multiscale collective effects in molecular and cell ensembles. It was shown that nonlinear behavior of biological systems can be associated with the generation of characteristic collective modes representing the open states in molecular and cell organization as the mechanism of the coherent expression dynamics. The mechanical DNA model is developed to study the nonlinear dynamics of the helicoidal geometry DNA molecule. To construct the model of DNA the PeyrardBishop-Barbi approach has been applied. The analytical small localized solutions as the discrete breather and the antikink have been obtained by multiple scale expansion method for multicomponent lattices. The set of collective open states (breathers) in the molecular ensembles provides the collective expression dynamics to attract cells toward a few preferred global states. This result allows the formulation of the experimental strategy to analyze the qualitative changes in cell dynamics induced by mentioned collective modes. The biomechanical changes have been shown experimentally using the original data of Coherent Phase Microscopy analyzing the time series of phase thickness fluctuations. Study of the mechanical aspects of the behavior of single cells is a prerequisite for the understanding of cell functions in the case of qualitative changes in diseases affecting the properties of cells and tissues morphology to develop diagnostic and treatment design methodology.
\end{abstract}

Key words: DNA, Peyrard-Bishop, helicoidal model, open states, modulation interference microscopy.

\section{INTRODUCTION}

Along with the application of research, taking into account the change in the mechanical properties of biological objects (e.g., vascular cardiology), currently, considerable attention is attracted to the study of changes in the mechanical behavior of bio-molecules and cells in the development of cancer, in which qualitative differences are installed in the mechanical properties of normal and cancer cells manifested at the tissue level, including the development of tumors.

Changing the mechanical responses of cells and tissues are potentially reflects the degree of damage of the biological system and can be used as a new diagnostic tool to complement traditional biochemical and molecular genetic methods currently used in clinical practice. The prospect of using the results of mechanobiology for classification of diseases related to mechanical changes of cells and tissues, suggests study of well-defined and standardized methods and protocols for data collection and processing. Direct research of mechanobiology

\footnotetext{
*nas@icmm.ru

†elena.a.korznikova@gmail.com

dmitriev.sergey.v@gmail.com

§naimark@icmm.ru
} 
responses of cells by coherent phase microscopy, tissue by the analyzing temperature fluctuations with the following definition of meaningful collective degrees of freedom allows the determination of dynamic stability of biological systems and their qualitative changes with damage accumulation.

Study of nonlinear dynamics of DNA molecule attracted interest of biologists and physicists as the key factor to link physical and biological properties of this biological crystal in the attempt to highlight the evolution of biological systems including the levels of cells and tissue. DNA belongs to the class of biopolymers and has an important biological function, which is the ability to store and transmit genetic information. It is a complex dynamic system consisting of many atoms and has a quasi-one-dimensional structure with specific symmetry, multiple degrees of freedom, and many types of movements and a special distribution of internal interactions [1]. These functions of DNA are discussed in some DNA models to investigate the relationship of structural-dynamic and functional properties of the molecule [2-13]. A comprehensive review on the DNA properties can be found in the work [14]. Various nonlinear excitations have been analyzed in DNA [8-17] in relation to charge transfer [8-12] or discrete breather excitation by external factors [13]. However, most of these studies do not take into account simultaneously the helicoidal geometry of DNA, damping effect and influence of external force.

Numerous researches have been devoted to the study of both the viscous dissipation effect and external force acting on DNA or the topological constraints to the helicoidal structure of the molecule. For instance, research by Sulaiman et al. [18] has used the Caldirola-Kanai approach [19] for the Peyrard-Bishop model [20] to consider the damping effect and external force. It was shown that the presence of viscosity is decelerated DNA breathing and on other hand the external force accelerates the breather propagation. Barbi et al. [21, 22] modified the Peyrard-Bishop model to take into account helicoidal geometry of DNA molecule. The solitonic solution was obtained for the system consisting of nucleotides with two degrees of freedom (for radial one it was the breather, for angular one it was the kink).

New model of DNA is presented, which considers the helicoidal shape of DNA molecule taking into account the damping effect and external force. It is based on applying the Caldirola-Kanai approach for the Peyrard-Bishop-Barbi model to develop the motion equations for nucleotides.

The paper is organized as follows. In Section 2 DNA structure and the stabilizing forces are described. Description of the DNA helicoidal model is given in Section 3. Mathematical formulation of the model and an approximate solution to this model are presented in Section 4. The Hamilton function of the model under consideration is explained in Section 4. The role of breather modes is described in Section 5. In Section 6 we talk about expression domains as coherent open states. The results of laser modulation interference microscopy are presented in Section 7. Section 8 concludes the paper.

\section{DNA STRUCTURE AND STABILIZING FORCES}

DNA consists of two polymer chains twisted around a common axis to form a double helix. The polymer chain is formed from monomers that are called nucleotides. A nucleotide consists of a phosphate group, a sugar ring and a nitrogenous base (adenine, thymine, guanine, and cytosine). Nucleotides connect either with the sugar phosphate backbone or a nitrogen base.

Two polynucleotide chains are held together by hydrogen bonds between the bases. The hydrogen bases combined in pairs according to complementary principle. The forces stabilizing the structure of DNA include stacking interactions between adjacent bases along the DNA axis and provide long-range interactions inside and outside the sugar phosphate backbone (in particular covalent bonds). 


\section{HELICOIDAL MODEL OF DNA WITH DAMPING AND EXTERNAL FORCE}

The helicoidal model has been proposed by Barbi et al. [21] to consider the helicoidal structure of the DNA molecule, base-base interactions in a pair and along the sugar-phosphate backbone coupling. Denaturation [21] and melting transition [22] have been investigated by the model.

We propose a modification of the helicoidal model of the DNA molecule, taking into account the effect of energy dissipation and external force. It is assumed that DNA consists of a sequence of $N$ nucleotide pairs. The group consisting of a sugar ring and the associated base is treated as a point mass (without distinction between different types of bases). The phosphate backbone between the two base pairs is modeled as an elastic rod. The point masses within the pair move symmetrically about the axis of the molecule. Each point mass has two degrees of freedom: radial and angular. The radial degree of freedom is determined by the parameter $r_{n}$ (distance between the center of symmetry and the point mass). Angular degree of freedom - angle $\varphi_{n}$, which is defined as the angle formed between the axis connecting the point masses inside the pair and the reference direction. Pairs of point masses move in parallel planes, the distance between them is fixed $h=3.4 \AA$ (Fig. 1). Rotation is defined as the difference between the angles of adjacent point masses $\theta_{n}=\varphi_{n}-\varphi_{n-1}$ (Fig. 1). It is assumed that the DNA molecule is in-form, with the following geometric parameters $R_{0}=10 \AA, \theta_{0}=36^{\circ}$ (Fig. 1). The object of modeling is considered in the framework of nonlinear dynamics, because DNA is a dynamic object. The energy of the system is determined by the Hamiltonian. As the potential describing the interaction between point masses within the pair (which corresponds to the hydrogen bond between the bases), the Morse potential is chosen because it describes the interaction between atoms and molecules quite accurately. It is assumed that the relationship between adjacent point masses can be described by an elastic potential that considers the relationship between the radial and angular degrees of freedom. In the considered model dissipation is taken into account in a specific way that allows Hamiltonian formulation. This enables the use of various techniques developed for the Hamiltonian systems even though the considered model includes the viscosity and external force. This is achieved through the energy balance between the kinetic energy, potential energy and the energy from the external force in a way that the kinetic energy of the system decreases with time due to dissipation, while the potential energy and the energy from internal force increase accordingly. The rate of decrease and increase depends on the dissipation factor $\gamma$ [18]. The system operates in an external conservative force $F$ which can mimic forces of mechanical or chemical origin. Other external influences on the system are neglected.

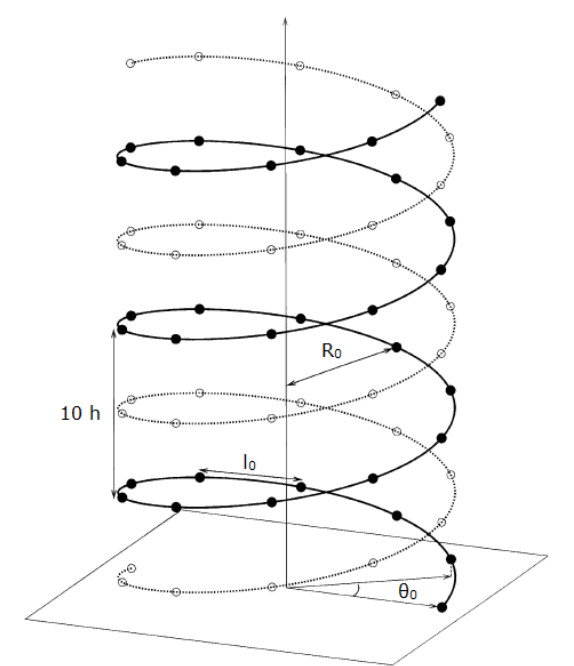

Fig. 1. Schematic view of the mechanical analogue of the DNA molecule [23]. 
Thus, the parameters describing the simulated system are the distance between the center of symmetry and the $n$-th point mass $r_{n}$ and the rotation of the $n$-th point mass relative to $(n-1)$-th $\theta_{n}$. To determine the parameters of the model at any time it is necessary to solve the system of equations of motion of point masses, determined from the Hamilton function.

\section{EQUATIONS OF MOTION OF THE DNA NUCLEOTIDES AND THEIR APPROXIMATE SOLUTION}

The behavior of the modeling object is described by the Hamilton function

$$
H\left(p_{r}, p_{\varphi}, r_{n}, \varphi_{n}\right)=e^{-\gamma t} E_{\text {kin }}\left(p_{r}, p_{\varphi}\right)+e^{\gamma t} U\left(r_{n}, \varphi_{n}\right)-e^{\gamma t} E_{F},
$$

where $E_{\text {kin }}$ is the kinetic energy of the system, $U$ is the potential energy of the system, $E_{F}$ is the energy entering the system from an external source, $p_{r}$ is the momentum of the point mass in a radial direction, $p_{\varphi}$ the angular momentum of a point mass in the angular direction, $\gamma$ is the damping constant.

The potential energy of the system is given as

$$
U=U_{\text {hyd }}+U_{\text {cov }}+U_{\text {backbone }},
$$

where $U_{\text {hyd }}$ is the energy of hydrogen bonds between bases within one pair of nucleotides, $U_{\text {cov }}$ is the energy of covalent bonds of nucleotides stacked one above the other, $U_{\text {backbone }}$ is the energy of sugar phosphate bonds responsible for the preservation of the helicoidal shape of the DNA molecule.

The energy of hydrogen bonds between the bases within one pair of nucleotides is described by the Morse potential, which has the following form

$$
U_{\text {hyd }}=\sum_{n} \frac{D}{2}\left(e^{-\alpha\left(r_{n}-R_{0}\right)}-1\right)^{2},
$$

where $D$ and $\alpha$ are the depth and the width of the potential hole respectively.

We introduce a relation describing the covalent bond arising in the sugar phosphate backbone. Since the covalent bond is much stronger than the hydrogen bond, it can be taken as a harmonic approximation

$$
U_{\mathrm{cov}}=\sum_{n} \frac{K}{2}\left(l_{n-1, n}-l_{0}\right)^{2},
$$

where $K$ is the stiffness coefficient of the covalent bonds, $l_{n-1, n}$ is the actual distance between $n-1$ and $n$ points mass, $l_{0}$ is the equilibrium distance between $n-1$ and $n$ points mass.

Since the energy of covalent bonds (4) tends to a minimum for arbitrarily oriented pairs of point masses, it is necessary to introduce an additional potential that determines the correct helix geometry of the simulated system

$$
U_{\text {backbone }}=\sum_{n} \frac{G_{0}}{2}\left(\varphi_{n+1}+\varphi_{n-1}-2 \varphi_{n}\right)^{2},
$$

where $G_{0}$ is the stiffness coefficient of the sugar phosphate backbone. This potential does not contribute to the total energy of the system (vanishes) only when the base pairs form a spiral.

Substituting (2), (3), (4) and (5) into (1) yields the final Hamiltonian 


$$
\begin{aligned}
& H=e^{-\gamma t} \sum_{n} \frac{m}{2}\left(\left(\frac{p_{r}}{2 m}\right)^{2}+\left(\frac{p_{\varphi}}{2 m r_{n}}\right)^{2}\right)+e^{\gamma t}\left(\sum_{n} \frac{D}{2}\left(e^{-\alpha\left(r_{n}-R_{0}\right)}-1\right)^{2}+\sum_{n} \frac{K}{2}\left(l_{n-1, n}-l\right)^{2}\right) \\
& +e^{\gamma t}\left(\sum_{n} \frac{G_{0}}{2}\left(\varphi_{n+1}+\varphi_{n-1}-2 \varphi_{n}\right)^{2}-\sum_{n} F_{n}(t)\left(r_{n}+r_{n} \varphi_{n}\right)\right),
\end{aligned}
$$

where $m$ is the nucleotide mass, $F_{n}(t)$ is an external stochastic force.

For the convenience of studying the model, the dimensionless Hamilton function is obtained. To do this, we introduce the parameters $Y_{n}$ and $\Phi_{n}$ denoting the displacement of the point mass relative to the equilibrium state in the radial and angular directions, respectively

$$
\begin{gathered}
Y_{n}=r_{n}-R_{0}, \\
\Phi_{n}=\varphi_{n}-n \theta_{0} .
\end{gathered}
$$

A limit is introduced on the amplitude of displacements of point masses in the radial $Y_{n} \ll<1$ and the angular $\Phi_{n}<<1$ directions. Then the Morse potential of $U_{\text {hyd }}$ and the potential of $U_{\text {cov }}$ can be written for small displacements in the following form

$$
\begin{gathered}
U_{\text {hyd }} \approx \frac{D}{2} \sum\left(\alpha^{2} Y_{n}^{2}-\frac{1}{2} \alpha^{3} Y_{n}^{3}+\frac{7}{8} \alpha^{4} Y_{n}^{4}\right), \\
U_{\mathrm{cov}} \approx \frac{K}{2} \sum_{n} \frac{R_{0}^{4} \sin ^{2} \theta_{0}}{l_{0}^{2}}\left(\Phi_{n}-\Phi_{n-1}\right)^{2} \\
+\frac{K}{2} \sum_{n} \frac{R_{0}^{2}\left(1-\cos \theta_{0}\right)^{2}}{l_{0}^{2}}\left(Y_{n}+Y_{n-1}\right)^{2} \\
+\frac{K}{2} \sum_{n} \frac{2 R_{0}^{3}\left(1-\cos \theta_{0}\right) \sin \theta_{0}}{l_{0}^{2}}\left(\Phi_{n}-\Phi_{n-1}\right)\left(Y_{n}+Y_{n-1}\right) .
\end{gathered}
$$

We introduce the dimensionless quantities

$$
\begin{aligned}
& y_{n}=\alpha Y_{n}, \phi_{n}=\alpha R_{0} \Phi_{n}, K \rightarrow K / D \alpha^{2}, \\
& R_{0} \rightarrow R_{0} \alpha, h \rightarrow h \alpha, G_{0} \rightarrow G_{0} / D, l_{0} \rightarrow \alpha l_{0}, \\
& \gamma \rightarrow \gamma \sqrt{m / D \alpha^{2}}, t \rightarrow t \sqrt{D \alpha^{2} / m}, \\
& H \rightarrow H / D .
\end{aligned}
$$

As result we have the dimensionless Hamiltonian

$$
\begin{aligned}
& H=e^{-\gamma t} \sum_{n} \frac{1}{2}\left(p_{y}^{2}+\frac{p_{\phi}^{2}}{\left(1+y_{n} / R_{0}\right)^{2}}\right)+e^{\gamma t}\left(\sum_{n} \frac{1}{2}\left(y_{n}^{2}-\frac{1}{2} y_{n}^{3}+\frac{7}{8} y_{n}^{4}\right)+\sum_{n} \frac{K_{y y}}{2}\left(y_{n}+y_{n-1}\right)^{2}\right) \\
& +e^{\gamma t}\left(\sum_{n} \frac{K_{y \phi}}{2}\left(y_{n}+y_{n-1}\right)\left(\phi_{n}-\phi_{n-1}\right)+\sum_{n} \frac{K_{\phi \phi}}{2}\left(\phi_{n}-\phi_{n-1}\right)^{2}+\sum_{n} \frac{G_{0}}{2}\left(\phi_{n+1}+\phi_{n-1}-2 \phi_{n}\right)^{2}\right) \\
& -e^{\gamma t} \sum_{n} C F_{n}(t)\left(y_{n}+R_{0}\right)\left(1+\left(\phi_{n} / R_{0}+n \theta_{0}\right)\right),
\end{aligned}
$$

where 


$$
\begin{aligned}
& K_{y y}=\frac{K R_{0}^{2}}{D \alpha^{2} l_{0}^{2}}\left(1-\cos \theta_{0}\right)^{2}, \\
& K_{\phi \phi}=\frac{K R_{0}^{2}}{D \alpha^{2} l_{0}^{2}} \sin ^{2} \theta_{0}, \\
& K_{y \phi}=\frac{K R_{0}^{2}}{D \alpha^{2} l_{0}^{2}} \sin \theta_{0}\left(1-\cos \theta_{0}\right), \\
& G=\frac{G_{0}}{D \alpha^{2} R_{0}^{2}}, C=\frac{1}{D \alpha} .
\end{aligned}
$$

From the Hamiltonian in (12), one can obtain the canonical coordinates,

$$
\begin{gathered}
\dot{y}_{n}=\frac{\partial H}{\partial p_{y}}, \\
\dot{\phi}_{n}=\frac{\partial H}{\partial p_{\phi}}, \\
\dot{p}_{y}=-\frac{\partial H}{\partial y_{n}}, \\
\dot{p}_{\phi}=-\frac{\partial H}{\partial \phi_{n}} .
\end{gathered}
$$

Substituting (14) and (15) into (16) and (17), respectively, yields a system of differential equations with the initial conditions

$$
\left\{\begin{aligned}
& \ddot{y}_{n}=e^{-2 \gamma t} \frac{2}{R_{0}} \frac{\dot{\phi}_{n}^{2}}{\left(1+y_{n} / R_{0}\right)}-2\left(y_{n}-\frac{3}{4} y_{n}^{2}+\frac{7}{4} y_{n}^{3}\right)-K_{y \phi}\left(\phi_{n+1}-\phi_{n-1}\right) \\
&-2 K_{y y}\left(y_{n-1}+2 y_{n}+y_{n+1}\right)-\gamma \dot{y}_{n}+2 C F_{n}(t)\left(1+\left(\phi_{n} / R_{0}+n \theta_{0}\right)\right) \\
& \ddot{\phi}_{n}=-\frac{2}{R_{0}} \dot{y}_{n} \dot{\phi}_{n}-\frac{2}{R_{0}} y_{n} \ddot{\phi}_{n}-\frac{2}{R_{0}^{2}} y_{n} \dot{y}_{n} \dot{\phi}_{n}-\frac{1}{R_{0}^{2}} y_{n}^{2} \ddot{\phi}_{n}+K_{y \phi}\left(y_{n+1}-y_{n-1}\right) \\
&+2 K_{\phi \phi}\left(\phi_{n+1}+\phi_{n-1}-2 \phi_{n}\right)-2 G_{0}\left(\phi_{n+2}+\phi_{n-2}-4 \phi_{n+1}-4 \phi_{n-1}+6 \phi_{n}\right) \\
&-\gamma \dot{\phi}_{n}-\frac{2}{R_{0}} \gamma y_{n} \dot{\phi}_{n}-\frac{1}{R_{0}^{2}} \gamma y_{n}^{2} \dot{\phi}_{n}+\frac{2}{R_{0}} C F_{n}(t)\left(y_{n}+R_{0}\right) \\
& y_{n}(0)=Y_{0}, \phi_{n}(0)=\Phi_{0}
\end{aligned}\right.
$$

In order to look for approximate localized solution of the equations of motion of the DNA nucleotides, we use the multiple scale technique for multicomponent lattices [21]. The method amounts to looking for wave-packet like solution. At the first step of the method, we determine the carrier vector as a phonon mode of the linearized system, increasing progressively the space and time scales. Next, we deduce the partial differential equation that identifies the envelope velocity with the wave-packet group velocity. Finally, we derive the nonlinear Schrodinger equation for the envelope, whose diffusion coefficient is in fact the wave packet group velocity dispersion.

Figure 2 shows analytical solution of the system (18) in displacement and time variable, as function of base pairs and time, obtained by the multiple scale technique for multicomponent lattices. The approximate localized solution of the equations of motion of the DNA nucleotides derived with the following model parameters $D=0.04 \mathrm{eV}, \alpha=4.45 \AA^{-1}$, 
$K=0.04 \mathrm{eV} \AA^{-2}, G_{0}=0.5 K R_{0}^{2}, m=300$ a.m.u., $\gamma=0.05 \mathrm{~kg} / \mathrm{s}, F=15 \mathrm{pN}$. The radial motion has the shape of discrete breather (Fig. 2,a) and angular motion exhibits an antikink structure (Fig. 2,b). Also, according to the results, we can conclude that the presence of viscosity decelerated DNA breathing, and the influence of the external force accelerates the breather propagation. This result is consistent with the study [8].

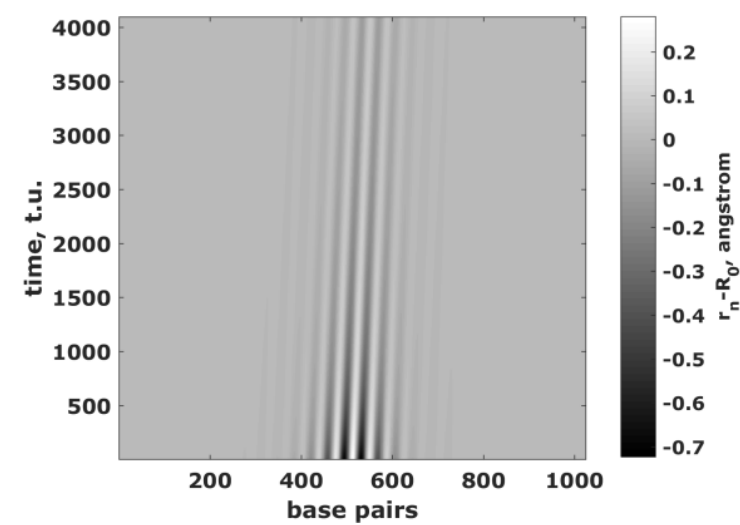

(a)

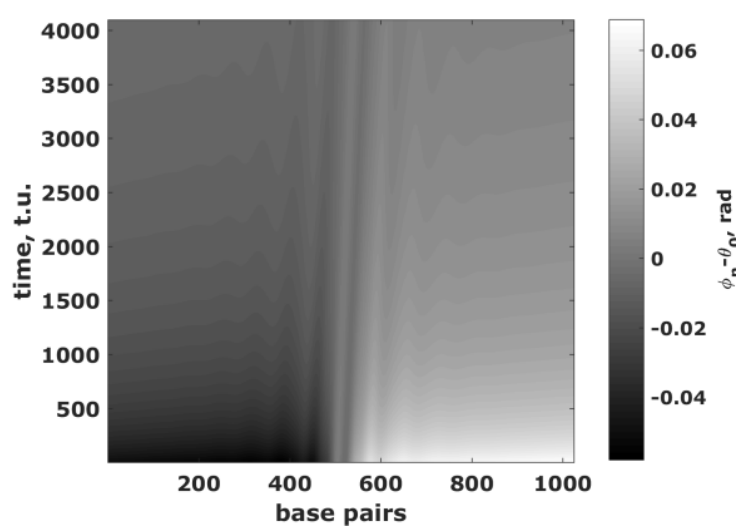

(b)

Fig. 2. Analytical solution of the DNA helicoidal model with the damping and constant external force. The radial displacement $r_{n}(t)-R_{0}$ (a). The corresponding angular displacement (b). The chain length is of 1024 cites. The total time is 4096 t.u.

\section{BREATHER MODES AND GENE EXPRESSION CRITICALITY}

The formation of multiscale open states can be considered as the controlling factor for collective gene expression DNA modes. The living cells are considered as the ensemble of a large number of interacting molecular species (DNA, RNA, proteins, and metabolites) providing the precise response to environmental stimuli due to the selection of specific pathways (differentiation, immune response). This robust organization in cells can be linked to the open state expression mechanisms of thousands of genes coordinated by a few key transcription factors $[24,25]$. These mechanisms are related to attractor states according to open complex (gene-expression) landscape [26, 27] providing the phenotypic states. The attractor concept envisages the system as evolving toward a preferred (minimal energy) state (called an attractor set) due to appearance of 'globally convergent' solutions. These solutions attract the system dynamics in the presence of stochastic fluctuations related to a gene-bygene interaction. Attractor states can be realized in the presence of a rugged non-equilibrium free energy landscape in the terms of corresponding variables. A fundamental question was articulated in [28] concerning the problem of cell dynamics controlling genome-wide expression: What is the driving force that attracts the entire system toward a few preferred global states, thus making the genome act as a single integrated system?

\section{EXPRESSION DOMAINS AS COHERENT OPEN STATES}

The criticality model of an early response to growth factors in a MCF-7 breast cancer cell population was proposed in [29], that characterizes distinct expression domains: dynamic, transit and static domains according to the degree of temporal variation in expression was analyzed in term of mean field (averaging) behavior of mRNAs based on the temporal expression changes. Self-similar unimodal-bimodal transition of the whole expression was established for the density profile of an ensemble of mRNA expression. These singular and scaling behaviors identify the transition as the expression phase transition driven by selforganized criticality (SOC). It was found that temporal development of criticality leads to autonomous bistable switch for each domain providing the coherent expression states. Criticality of the whole expression of MCF-7 cell stimulated by heregulin (HRG) exhibits 
three distinct response domains going from higher to lower normalized root-mean-squarefluctuation (nrmsf): dynamic domain; unimodal profile, (middle) transit domain. The energy profiles correspond to free energy in terms of the symmetry argument of Landau. The temporal invariant flatness of energy profile suggests the existence of the critical point, where the change in expression between different time points is around zero. A critical (from unimodal to bimodal) behavior corresponds to a global phase transition in the whole gene expression profile with shift in the frequency profiles of the ensemble of stochastic mRNA expression due to flattening of the unimodal. According to these results the critical dynamics of gene expression show the singularity induced scaling, that is characteristic for selforganized criticality (SOC). The single expression shows a scattered stochastic expression distribution [3]. It was concluded that a single gene level is not the correct scale where real global genome response corresponds to the SOC nature.

The transformation landscape for both mRNA expression and open states has pronounced features of the free energy flattening corresponding to the transition from the unimodal-tobimodal free energy profiles at some critical points $\delta_{*}$ and $\delta_{c}$ for internal variable $\delta$ that represents the current susceptibility of the system to initiate the open modes. This internal variable $\delta$ is the ratio of the mean size of current open modes and the spacing between open modes [2]. The transitions over the critical points $\delta_{*}$ and $\delta_{c}$ lead to the qualitative change of the free energy metastability and the generation of collective open modes with breathers, solitary waves and blow-up dynamics. Transition from breather to solitary and blow-up dynamics is realized according to the universal scenario (structural-scaling transition) as the subjection of the open complex (gene expression) dynamics to the sequence of corresponding self-similar solutions - the eigen-forms of nonlinear out-of-equilibrium system. This transition can be considered as possible scenario of the DNA and cell localized plasticity leading to the anomalous DNA and cell softening (localized damage).

These qualitative different eigen-forms (self-similar solutions) for open complex variables represent the set of collective order parameters, which subject the system dynamics to follow the $\delta$ kinetics with typical signs of SOC scenario. The $\delta$ kinetics could be considered as natural epigenetic driving force controlling DNA and cell dynamics. The presence in the DNA ensemble the qualitative different collective modes (attractor states) could be associated with fluctuations of some experimentally measured parameters (for instance, genome-wide expression) and interpreted as the morphological dynamic pattern with the signs of multifractality. Degeneration of this dynamics into the blow-up controlled dynamics can be recognized in fluctuation analysis as the transition from multi- to monofractality.

\section{LASER MODULATION INTERFERENCE MICROSCOPY. CONVERGENT COHERENT STATES}

The biomechanical changes are important in the case of cancer, which has been shown experimentally that the change in the stiffness of cells is a hallmark of cancer. Thus, an understanding of the mechanical aspects of the behavior of single cells is a prerequisite for the understanding of cell functions in the case of qualitative changes in diseases affecting the properties of tissue morphology at the level of cells and tissues, which allow the use of diagnostic and treatment design methodology. Study of nonlinear dynamics of cell and cytoskeleton structures, objectification of cytological diagnosis of cancer (morphometry) were conducted using the original data of modulation interference microscopy analyzing the time series of phase thickness fluctuations [30-32] in the cross-sections of the nucleus, the nucleolus, cytoplasm. The method for the estimation of spatial temporal invariants (in terms of the Hurst scaling exponent) allowed the demonstration of the links of temporal correlations of finite-amplitude phase thickness fluctuation in terms of multi- and monofractality with the states of the normal and cancerous cells [33-35]. The MIM data correspond to three types of 
patterns: cell phase image (Fig. 3,a), the track diagram as the image of equal phase thickness (Fig. 3,b) and the track diagram as the surface thickness image (Fig. 3,c).

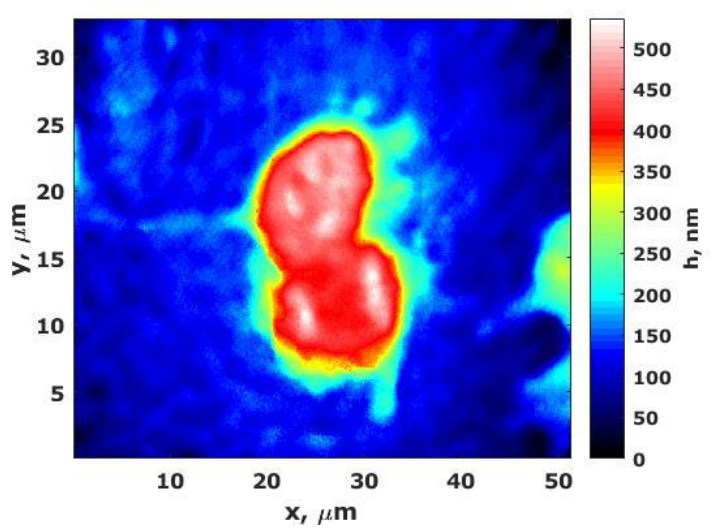

(a)

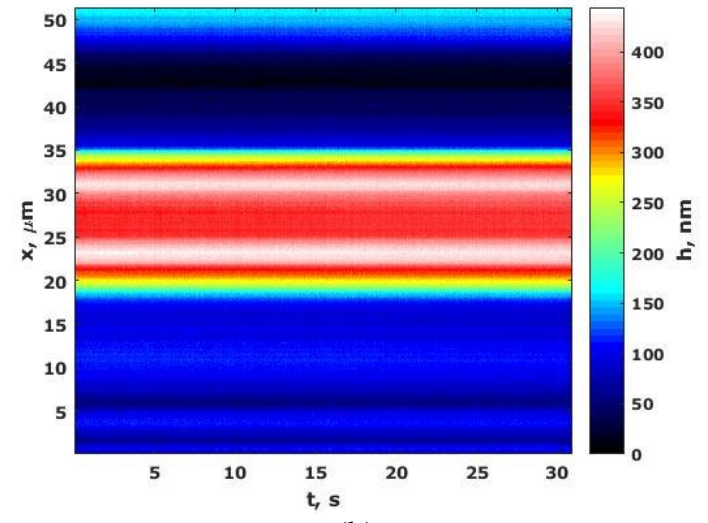

(b)

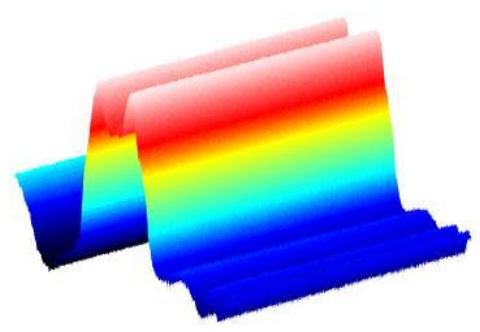

(c)

Fig. 3. Modulation interference microscopy data: three types patterns of the cell image (a), 2D (b) and 3D (c) track diagram.

Registration of MIM pattern high spatial and temporal resolution allowed the analysis of dynamic processes in living cells. Biomaterial for the MIM study was prepared by the Herzen Moscow Oncology Research Institute. 22 cancer cells and 22 healthy cells from intact organs were analyzed as MIM topograms (44 images) and 88 track diagrams (Fig. 4, 5). Optically dense area corresponds to the cell nuclei, and MIM data were used to study under the life dynamics of cell nucleolus. MIM data in the nucleolus area of cancer cell is presented as the phase thickness dynamics in time (Fig. 6).
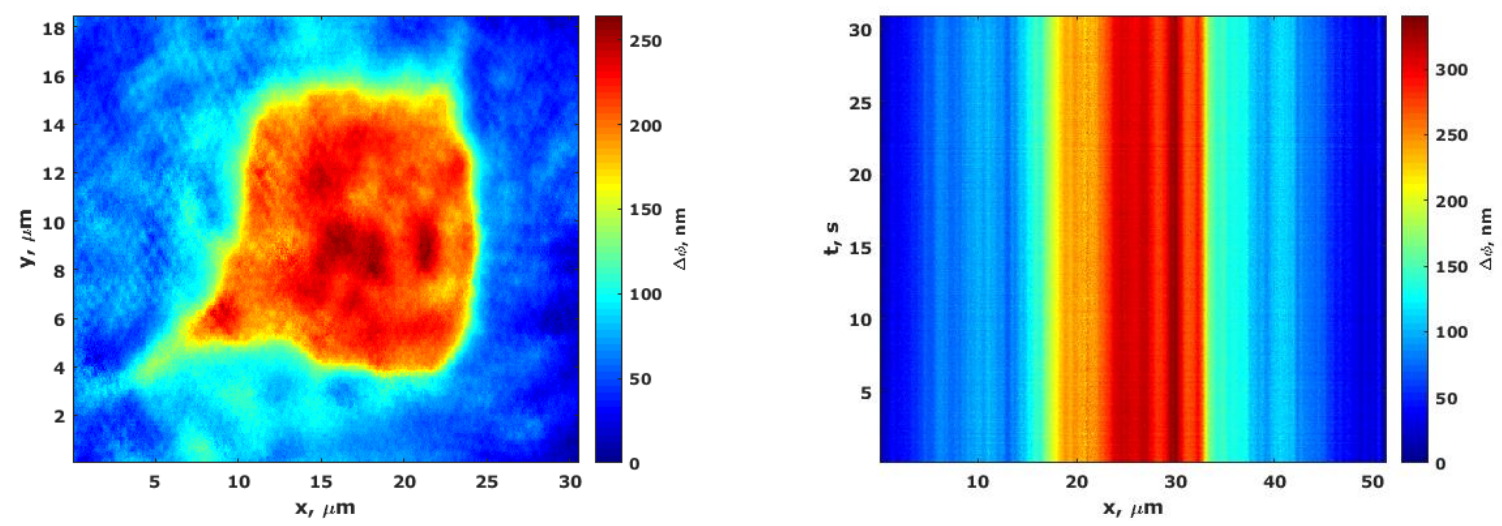

Fig. 4. Topogram (left) and the track diagram (right) of the drug culture of MCF-7 cancer cells. 

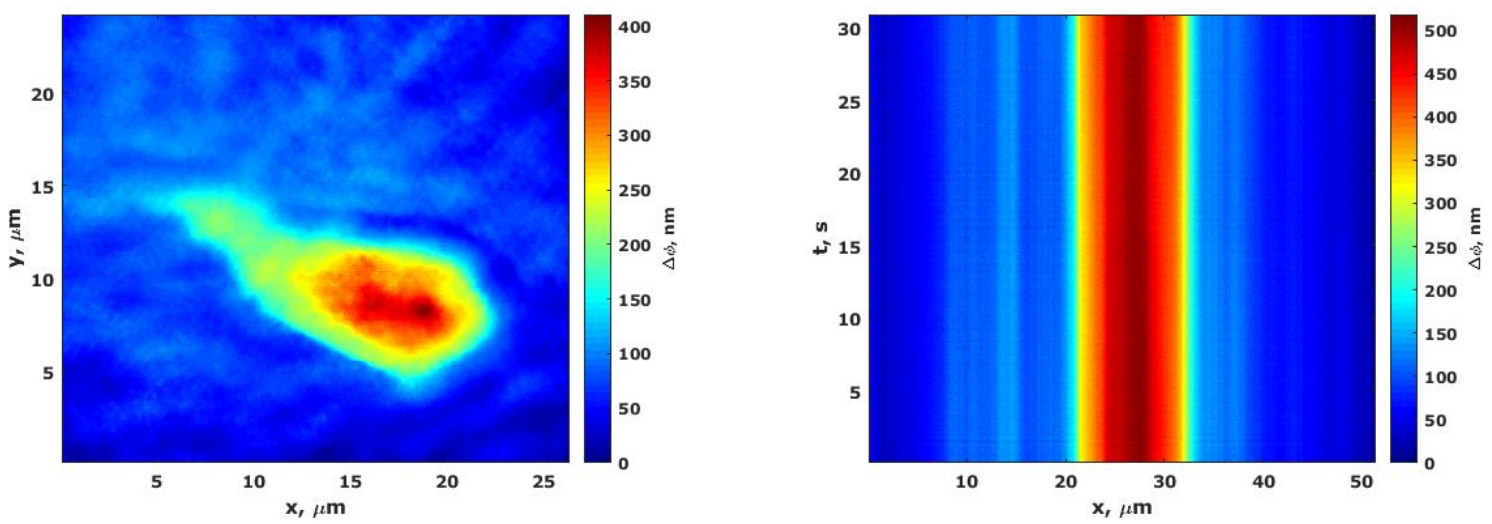

Fig. 5. Topogram (top) and the track diagram (bottom) of the drug HEK 293 healthy cells.

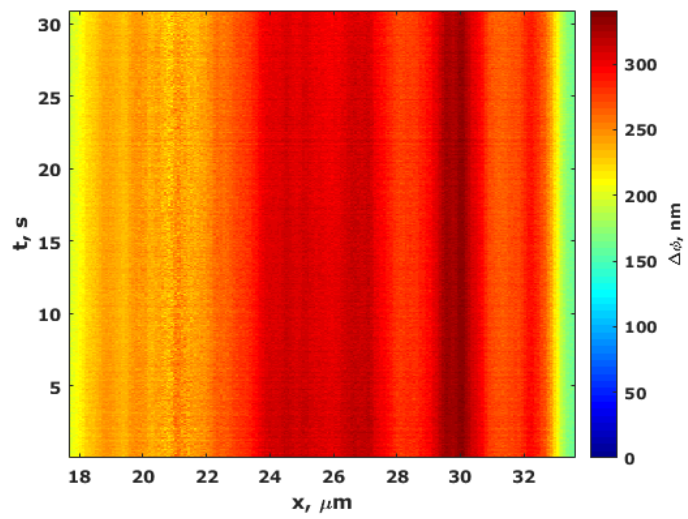

Fig. 6. Phase thickness dynamics in nucleolus area of cancer cell.

The estimation of spatial temporal invariants (in terms the Hurst scaling exponent) allowed the demonstration of the links of temporal correlations of finite-amplitude phase thickness fluctuation with multi- and monofractal states of the normal and cancerous cells.

\section{CONCLUSIONS}

The main objective of the present work was to propose a new model of DNA which considers its helicoidal shape, the damping and external force. Current models take into account either the viscous dissipation effect and external force acting on DNA or the topological constraints to the helicoidal structure of the molecule. To solve this problem, two approaches were used together: the Peyrard-Bishop-Barbi model and the Caldirola-Kanai approach.

We derived the dissipative term and the external force term of equations of motion of the DNA nucleotides directly from the Hamilton function. The main advantage of this approach is able to calculate the thermodynamic behaviors of the system under consideration for future studies. Future research will concentrate on the looking for approximate localized solution at different types of external force and different values of the damping constant and their following investigation.

The mechanical response of biomolecules and cells is very complex due to non-linear, non-homogeneous, non-isotropic and viscous behavior. In most cases, models employed for describing mechanical behavior or analyzing mechanical data need to be sufficiently simplified to avoid many not well-defined or only phenomenological derived parameters.

One of the key problems that will be improved to understand molecular, cellular, and architectural changes at the tissue level can be linked to the spatial-temporal analysis of signals extracted by molecular-genetic study. Molecular-morphological signs of oncogenesis could be linked to multiscale collective effects in molecular, cell and tissue dynamics, the 
development of methods characterizing the mechanisms of transformation, development of experimental approaches based on the estimation of spatial-temporal invariants using quantitative morphology data.

\section{ACKNOWLEDGEMENTS}

The work was financially supported by the Russian Federation via the Ministry of Science and Higher Education of the Russian Federation (project identifier RFMEFI60718X0202).

\section{REFERENCES}

1. Yakushevich L.V. Nonlinear physics of DNA. Weinheim: WILEY-VCH Verlag GmbH \& Co. KGaA., 2004. 205 p.

2. Naimark O.B. Structural-scaling transitions and localized distortion modes in the DNA double helix. Phys. Mesomech. 2007. V. 10. No. 1-2. P. 33-45.

3. Dauxois T., Peyrard M., Bishop A.R. Entropy-driven DNA denaturation. Phys. Rev. E. 1993. V. 47. No. 1. P. 44-47.

4. Campa A. Bubble propagation in a helicoidal molecular chain Phys. Rev. E. 2001. V. 63. No. 2. P. 021901.

5. Alvarez A., Romero F.R., Archilla J.F.R., Cuevas J., Larsen P.V. Breather tapping and breather transmission in a DNA model with an interface. Eur. Phys. J. B. 2006. V. 51. No. 1. P. 119-130.

6. Yakushevich L.V., Grinevich A.A., Ryasik A.A. Simulation of a kink movement in homogeneous and heterogeneous DNA sequences taking into account the dissipation. Russ. J. Numer. Anal. Math. Modelling. 2014. V. 29. No. 3. P. 197-204.

7. Zdravkovic S., Sataric M., Tuszynskic J. Biophysical Implications of the PeyrardBishop-Dauxois Model of DNA Dynamics. J. Comput. Theor. Nanosci. 2004. V. 1. No. 2. P. 169-179.

8. Lakhno V.D. DNA nanobioelectronics. International Journal of Quantum Chemistry. 2008. V. 108. No. 11. P. 1970-1981.

9. Lakhno V.D. Soliton-like solutions and electron transfer in DNA. Journal of Biological Physics. 2000. V. 26. No. 2. P. 133-147.

10. Lakhno V.D., Fialko N.S. Hole mobility in a homogeneous nucleotide chain. JETP Letters. 2003. V. 78. No. 5. P. 336-338.

11. Chetverikov A.P., Sergeev K.S., Lakhno V.D. Trapping and transport of charges in DNA by mobile discrete breathers. Mathematical Biology and Bioinformatics. 2018. V. 13. No. 1. P. 1-12. doi: 10.17537/2018.13.1.

12. Lakhno V.D., Chetverikov A.P. Excitation of bubbles and breathers in DNA and their interaction with the charge carriers. Mathematical Biology and Bioinformatics. 2014. V. 9. V. 1. P. 4-19. doi: 10.17537/2014.9.4.

13. Chetverikov A.P., Sergeev K.S., Lakhno V.D. The excitation of mobile discrete breathers in DNA by initial disturbance of displacements or velocities of a few of adjacent nucleotide pairs. Mathematical Biology and Bioinformatics. 2017. V. 12. No. 2. P. 375-384. doi: 10.17537/2017.12.375.

14. Shigaev A.S., Ponomarev O.A., Lakhno V.D. Theoretical and experimental investigations of DNA open states. Mathematical Biology and Bioinformatics. 2018. V. 13. No. Suppl. P. t162-t267. doi: 10.17537/2018.13.t162.

15. Velarde M.G., Chetverikov A.P., Ebeling W., Dmitriev S.V., Lakhno V.D. From solitons to discrete breathers. European Physical Journal B. 2016. V. 89. No. 10. P. 233.

16. Velarde M.G., Chetverikov A.P., Ebeling W., Dmitriev S.V., Lakhno V.D. Wave motions along lattices with nonlinear on-site and inter-site potentials. Cooperation 
and/or competition leading to lattice Solitons and/or discrete breathers. Proceedings of the Estonian Academy of Sciences. 2015. V. 64. No. 3. P. 396-404.

17. Dmitriev S.V., Korznikova E.A., Baimova Y.A., Velarde M.G. Discrete breathers in crystals. Physics-Uspekhi. 2016. V. 59. No. 5. P. 446-461.

18. Sulaiman A., Zen F.P., Alatas H., Handoko L.T. Dynamics of DNA breathing in the Peyrard-Bishop model with damping and external force. Physica D. 2012. V. 241. P. 1640-1647.

19. Ikot A.N., Akpabio L.E., Akpan I.O., Umo M.I., Ituen E.E. Quantum damped mechanical oscillator. International Journal of Optics. 2010. V. 1. No. 5-6. P. 1-6.

20. Peyrard M. Nonlinear dynamics and statistical physics of DNA. Nonlinearity. 2004. V. 17. No. 2. P. 1-40.

21. Barbi M., Cocco S., Peyrard M. Helicoidal model for DNA opening. Physics Letters A. 1999. V. 253. P. 358-369.

22. Barbi M., Lepri S., Peyrard M., Theodorakopoulos N. Thermal denaturation of a helicoidal DNA model. Physical Review E. 2003. V. 68. No. 6. P. 061909

23. Argwal J., Henning D. Breather solutions of a nonlinear DNA model including a longitudinal degree of freedom. Physica A. 2003. V. 323. P. 519-533.

24. Klevecz R.R., Bolen J., Forrest G., Murray D.B. A genomewide oscillation in transcription gates DNA replication and cell cycle. Proc. Natl. Acad. Sci. 2004. V. 101. No. 5. P. 1200-1205.

25. Yoshikawa K. Field hypothesis on the self-regulation of gene expression. J. Biol. Phys. 2002. V. 28. No. 4. P. 701-712.

26. Huang S., Eishier G., Bar-Yam Y., Ingber D.E. Cell Fates as High-Dimensional Attractor States of a Complex Gene Regulatory Network. Phys. Rev. Lett. 2005. V. 94. P. 128701-128705.

27. Goldberg A., Allis C.D., Bernstein E. Epigenetics: A Landscape Takes Shape. Cell. 2007. V. 128. No. 4. P. 635-638.

28. Tsuchiya M., Giuliani A., Hashimoto M., Erenpreisa J., Yoshikawa K. Emergent SelfOrganized Criticality in gene expression dynamics: Temporal development of global phase transition revealed in a cancer cell line. PLOS ONE. 2015. V. 11. P. 1-33.

29. Tsuchiya M., Hashimoto M., Takenaka Y., Motoike I.N., Yoshikawa K. Global genetic response in a cancer cell: Self-organized coherent expression dynamics. PLOS ONE. 2014. V. 9. P. 1-33.

30. Tychinsky V. P., Kretushev A.V., Klemyashov I.V., Vyshenskaya T.V., Shtil A.A., Zatsepina O.V. Coherent phase microscopy, a novel approach to study the physiological state of the nucleolus. Doklady Biochemistry and Biophysics. 2005. V. 405. P. 432-436.

31. Lyapunova E., Nikituk A., Bayandin Y., Naimark O., Rianna C., Radmacher M. Passive microrheology of normal and cancer cells after ML7 treatment by atomic force microscopy. In: AIP Conference Proceedings "International Conference on Physics of Cancer: Interdisciplinary Problems and Clinical Applications (PC'16)”. 2016. V. 1760. No. 1. P. 020046.

32. Popescu G., Quantitative Phase Imaging of Cells and Tissues. McGraw-Hill biophotonics: McGraw-Hill, 2011. 362 p.

33. Martinez-Torres C., Berguiga L., Streppa L., Boyer-Provera E., Schaeffer L., Elezgaray J., Arneodo A., Argoul F. Diffraction phase microscopy: retrieving phase contours on living cells with a wavelet-based space-scale analysis. Journal of Biomedical Optics. 2014. V. 19. No. 3. P. 8-19.

34. Gerasimova E., Audit B., Roux S.-G., Khalil A., Argoul F., Naimark O., Arneodo A. Multifractal analysis of dynamic infrared imaging of breast cancer. Europhysics Letters. 2013. V. 104. P. 64001. 
35. Gerasimova E., Audit B., Roux S.-G., Khalil A., Gileva O., Argoul F., Naimark O., Arneodo A. Wavelet-based multifractal analysis of dynamic infrared thermograms to assist in early breast cancer diagnosis. Frontiers in Physiology. 2014. V. 5. P. 176.

Received 12.11.2018

Revised 25.03.2019

Published 11.04.2019. 